\title{
Accounting for Missing Values in Score-Driven Time-Varying Parameter Models ${ }^{\text {th }}$
}

\author{
André Lucas $^{\mathrm{a}}$, Anne Opschoor ${ }^{\mathrm{a}}$, Julia Schaumburg $^{\mathrm{a}}$ \\ ${ }^{a}$ Vrije Universiteit Amsterdam and Tinbergen Institute
}

\begin{abstract}
Two alternative perspectives on dealing with missing data in the context of the score-driven time-varying parameter models of Creal et al. (2013) and Harvey (2013) lead to precisely the same dynamic transition equations. This ties the score-driven approach theoretically to the Expectation-Maximization framework for dealing with missing values.
\end{abstract}

Keywords: generalized autoregressive score models, missing values, Expectation-Maximization.

JEL: $\mathrm{C} 53, \mathrm{C} 52$

\section{Introduction}

We address the issue of missing values in observation-driven time-varying parameter models. Such models are widely applied in the empirical economics and econometrics literature and include well-known examples such as the generalized autoregressive conditional heteroskedasticity (GARCH) model of Engle (1982) and Bollerslev (1986), the autoregressive conditional duration (ACD) model of Engle and Russell (1998), the multiplicative error model (MEM) of Engle and Gallo (2006), and many more. In this paper, we focus on the class of score-driven models as introduced and popularized by Creal et al. (2011, 2013) and Harvey (2013). Score-driven models encompass earlier well-known models such as the normal GARCH and ACD models, but also give rise to entirely new models, such as the mixed measurement dynamic factor model of Creal et al. (2014) for macro and credit cycles, the dynamic Nelson-Siegel model with time-varying shape parameter of Quaedvlieg and Schotman (2016) for long-term pension liability hedging, the time-varying copula model of Oh and Patton (2016) to model credit risk in high dimensions, and the matrix- $F$ dynamic model for multivariate realized covariance matrices of Opschoor et al. (2014).

A key property of observation-driven models in the classification of Cox (1981) is that they define the time-varying parameter, such as a mean or a volatility parameter, in terms of its own lags and a (possibly nonlinear) function of lagged observations. This directly poses a problem for updating the time-varying parameter if a missing value is encountered: if the observation is missing, the function of lagged variables cannot be evaluated and the dynamic parameter cannot be updated.

In this paper we show how score-driven models provide a natural way to include the non-missing part of the data into the updating mechanism, while accounting for the missing data in an intuitive and consistent way. We do so by discussing two alternative perspectives of the missing value problem in our current setting and by showing that the two perspectives lead to exactly the same solution. Under the first perspective, we exploit the special feature of score-driven models that the direction in which the dynamic parameter is updated, equals the derivative of the $\log$ of the conditional predictive density. If (part of) the data are missing, this predictive density simplifies to a marginal density for the observed data part and the

\footnotetext{
Schaumburg thanks the Dutch National Science Foundation (NWO, grant VENI451-15-022) for financial support. Corresponding author: André Lucas, FEWEB/FIN Vrije Universiteit, De Boelelaan 1105, 1081 HV Amsterdam. Phone +31 20 598 6039.

Email addresses: a.lucas@vu.nl (André Lucas), a.opschoor@vu.nl (Anne Opschoor), j.schaumburg@vu.nl (Julia Schaumburg)
} 
propagation mechanism for the time-varying parameter adapts automatically. This approach is for example used in Creal et al. (2014), where no further motivation was provided. A second perspective sets the missing value problem in the much wider statistical literature about the treatment of missing values; see for example Little and Rubin (2014) for a textbook review. This is also the typical context where the ExpectationMaximization (EM) approach of Dempster et al. (1977) is used for estimation rather than the classical likelihood framework. Though natural, there is as of yet no theory linking the statistical missing value literature to the updating mechanism in score-driven models.

As the main contribution in this paper we show that a score-driven approach based on the marginal predictive density produces exactly the same result as a score-driven approach based on the EM criterion. This result is an important step in motivating how to deal with missing values in the context of score-driven models as in, for instance, Creal et al. (2014). At the same time, it closes the gap between the literature on score-driven time series models and the classical statistical missing value and EM literature. While doing so, we also show how a score-driven approach may be devised outside the familiar conditional predictive density context of Creal et al. (2011, 2013). In particular, we the scores based on the EM criterion function to drive the time-varying parameter constitute a novel perspective on the usefulness of score-driven dynamic parameter models.

The rest of this paper proceeds as follows. Section 2 explains the issue of missing values in the context of the generalized autoregressive score (GAS) model of Creal et al. (2013). ${ }^{1}$ Section 3 sets up a score based approach in the EM set-up of Dempster et al. (1977) and proves our theoretical result.

\section{Missing-values and standard GAS models}

Let $x_{t}$ and $y_{t}$ denote two vectors of observations, characterized by the conditional density

$$
\left(x_{t}, y_{t}\right) \sim p\left(x_{t}, y_{t} \mid f_{t}\right)
$$

where $f_{t}$ is a time-varying parameter, such as the conditional means of $x_{t}$ and $y_{t}$, their conditional variances, their correlations, or other higher order features of the conditional distribution; see Creal et al. (2011, 2013) and Harvey and Luati (2014) for a range of applications. We consider $x_{t}$ and $y_{t}$ as scalars in the remainder of this paper, but all derivations also go through in a higher dimensional context. The conditioning set of $p\left(\cdot, \cdot \mid f_{t}\right)$ can be augmented with other predetermined information, such as further lags of $x_{t}$ and $y_{t}$. We assume that the dynamics for $f_{t}$ are given by the generalized autoregressive score dynamics

$$
f_{t+1}=\omega+\beta f_{t}+\alpha s_{t}
$$

where $\omega, \alpha$, and $\beta$ are static parameters that need to be estimated, and $s_{t}$ is a scaled score step. In this paper we consider different score steps, namely based on a joint density $\left(s_{t}^{\text {joint }}\right)$, on a marginal density $\left(s_{t}^{\text {marg }}\right)$, and on an EM objective function $\left(s_{t}^{\mathrm{EM}}\right)$. For the case of a fully observed data vector $\left(x_{t}, y_{t}\right)$ we can work with the joint density and obtain the scaled score

$$
s_{t}=s_{t}^{\text {joint }}=S_{t} \frac{\partial \log p\left(x_{t}, y_{t} \mid f_{t}\right)}{\partial f_{t}},
$$

where $S_{t}$ is a scaling matrix that may depend on $f_{t}$ itself and typically corrects for the curvature of the score, such as

$$
S_{t}=\mathrm{E}_{t-1}\left[\frac{\partial \log p\left(x_{t}, y_{t} \mid f_{t}\right)}{\partial f_{t}} \frac{\partial \log p\left(x_{t}, y_{t} \mid f_{t}\right)}{\partial f_{t}^{\prime}}\right]^{-1} .
$$

The score step in equation (2) increases the local model fit in the steepest ascent direction of the local likelihood contribution and has information theoretic optimality properties; see Blasques et al. (2015).

\footnotetext{
${ }^{1}$ See also Harvey (2013), who refers to these models as dynamic conditional score models, or gasmodel.com for a much more complete compendium of papers on score-driven (GAS) models.
} 
An important advantage of the score driven approach is that it is observation-driven in the classification of Cox (1981). This enables us to write down the likelihood function in analytic form via a prediction error decomposition and to estimate the model's static parameter $\theta \equiv(\omega, \beta, \alpha)$ by standard maximum likelihood.

A drawback of the observation-driven specification is that a problem occurs if $y_{t}$ (and/or $x_{t}$ ) is missing: the score $s_{t}$ can then not be computed, as it depends on both $y_{t}$ and $x_{t}$. Creal et al. (2014) solve this problem by arguing that the score should be taken of the conditional observation density at time $t$. If $y_{t}$ is missing, the observation density at time $t$ collapses to the marginal density $p\left(x_{t} \mid f_{t}\right)$ for $x_{t}$ and thus the score should be computed based on this marginal density, i.e., using $\partial \log p\left(x_{t} \mid f_{t}\right) / \partial f_{t}$. The reasoning of Creal et al. (2014) appears intuitive: if $y_{t}$ is missing, the only information about $f_{t}$ can be obtained from $x_{t}$ and therefore from its marginal conditional density, such that

$$
s_{t}=s_{t}^{\operatorname{marg}}=S_{t} \frac{\partial \log p\left(x_{t} \mid f_{t}\right)}{\partial f_{t}}
$$

A different line of argument, however, argues from a conditional expectations perspective. The reasoning is as follows. Consider a setting of a bivariate normal $\left(x_{t}, y_{t}\right)$ with time-varying means. If we know that $x_{t}$ and $y_{t}$ are strongly positively correlated, and if at time $t$ the variable $x_{t}$ is above its mean while $y_{t}$ is missing, we might infer that $y_{t}$ is also above its mean at time $t$ with high probability, and use this inferred information to also update the mean of $y_{t}$ upward. At first sight, this alternative approach appears to be in line with the EM perspective of Dempster et al. (1977) to account for missing observations. It contrasts, however, with the previously described approach based on the score of the marginal distribution of $x_{t}$. There the mean of $y_{t}$ would not receive an update signal as the score of the marginal density $p\left(x_{t} \mid f_{t}\right)$ with respect to the mean of $y_{t}$ would be zero, and consequently the time-varying mean of $y_{t}$ would mean-revert via the transition equations (1) and (3).

\section{EM score models and equivalence result}

To close the gap between the score approach based on marginal distributions and the EM-based perspective, we introduce a score-based approach based on the local EM objective function

$$
\log \tilde{p}\left(x_{t} \mid f_{t}\right):=\mathrm{E}\left[\log p\left(x_{t}, y_{t} \mid f_{t}\right) \mid x_{t}, f_{t}\right] .
$$

This is a novel perspective on score-based time-varying parameter modeling, where usually the score is taken of a predictive density in a likelihood framework. Creal et al. (2016) take a similar perspective in a Generalized Method of Moments (GMM) setting. Just as $p\left(x_{t} \mid f_{t}\right)$ is the local (marginal) likelihood contribution in a predictive density context, $\tilde{p}\left(x_{t} \mid f_{t}\right)$ is the local contribution to the criterion function in an EM set-up. In fact, if there are no missing data, the two approaches coincide and $p\left(\cdot \mid f_{t}\right)=\tilde{p}\left(\cdot \mid f_{t}\right)$.

Using the EM perspective, $f_{t}$ is propagated by the (scaled) score of the conditional expectation of the $\log$ likelihood contribution at time $t$, i.e.,

$$
s_{t}=s_{t}^{\mathrm{EM}}=S_{t} \frac{\partial \log \tilde{p}\left(x_{t} \mid f_{t}\right)}{\partial f_{t}} .
$$

We note that this new score type model model automatically deals with missing values in a way that is fully compatible with the EM framework. In fact, $f_{t}$ is updated by exploiting the full dependence structure between $x_{t}$ and $y_{t}$ as given by the joint density. Therefore, if $x_{t}$ and $y_{t}$ are highly correlated, this information is also taken into account.

The key question now is what are the differences between a transition equation for $f_{t}$ based on score steps from a likelihood perspective and marginal distributions $\left(s_{t}^{\mathrm{marg}}\right)$ and one based on the EM perspective $\left(s_{t}^{\mathrm{EM}}\right)$. Our key theoretical result is that there is no difference and that the two approaches are actually fully equivalent. We state this in the following theorem.

Theorem 1. If the density $p\left(x_{t}, y_{t} \mid f_{t}\right)$ is correctly specified and has an (absolute) continuous conditional density $p\left(y_{t} \mid x_{t}, f_{t}\right), s_{t}^{\mathrm{EM}}=s_{t}^{\mathrm{marg}}$. 
Proof. We note that $\partial \log p\left(x_{t}, y_{t} \mid f_{t}\right) / \partial f_{t}=\partial\left(\log p\left(y_{t} \mid x_{t}, f_{t}\right)+\log p\left(x_{t} \mid f_{t}\right)\right) / \partial f_{t}$, such that

$$
\frac{\partial \log \tilde{p}\left(x_{t} \mid f_{t}\right)}{\partial f_{t}}=\mathrm{E}\left[\frac{\partial \log p\left(y_{t} \mid x_{t}, f_{t}\right)}{\partial f_{t}} \mid x_{t}, f_{t}\right]+\frac{\partial \log p\left(x_{t} \mid f_{t}\right)}{\partial f_{t}}=\frac{\partial \log p\left(x_{t} \mid f_{t}\right)}{\partial f_{t}}
$$

because

$$
\begin{aligned}
\mathrm{E}\left[\frac{\partial \log p\left(y_{t} \mid x_{t}, f_{t}\right)}{\partial f_{t}} \mid x_{t}, f_{t}\right] & =\int \frac{\partial \log p\left(y_{t} \mid x_{t}, f_{t}\right)}{\partial f_{t}} p\left(y_{t} \mid x_{t}, f_{t}\right) \mathrm{d} y_{t}=\int \frac{\partial p\left(y_{t} \mid x_{t}, f_{t}\right)}{\partial f_{t}} \mathrm{~d} y_{t} \\
& =\frac{\partial}{\partial f_{t}} \int p\left(y_{t} \mid x_{t}, f_{t}\right) \mathrm{d} y_{t}=\frac{\partial}{\partial f_{t}} 1=0 .
\end{aligned}
$$

Example. For concreteness, consider the example of a bivariate normally distributed $\left(x_{t}, y_{t}\right)^{\prime}$ with mean $f_{t}=\left(f_{x, t}, f_{y, t}\right)^{\prime}$, variances $\sigma_{x x}$ and $\sigma_{y y}$, and covariance $\sigma_{x y}$. We obtain

$$
\begin{aligned}
\mathrm{E}\left[\frac{\partial \log p\left(y_{t}, x_{t} \mid f_{t}\right)}{\partial f_{t}} \mid x_{t}, f_{t}\right] & =\mathrm{E}\left[\left(\begin{array}{cc}
\sigma_{x x} & \sigma_{x y} \\
\sigma_{x y} & \sigma_{y y}
\end{array}\right)^{-1}\left(\begin{array}{c}
x_{t}-f_{x, t} \\
y_{t}-f_{y, t}
\end{array}\right) \mid x_{t}, f_{t}\right] \\
& =\left(\begin{array}{cc}
\sigma_{x x} & \sigma_{x y} \\
\sigma_{x y} & \sigma_{y y}
\end{array}\right)^{-1}\left(\begin{array}{c}
\sigma_{x x} \sigma_{x x}^{-1}\left(x_{t}-f_{x, t}\right) \\
\sigma_{x y} \sigma_{x x}^{-1}\left(x_{t}-f_{x, t}\right)
\end{array}\right)=\left(\begin{array}{c}
\left(x_{t}-f_{x, t}\right) / \sigma_{x x} \\
0
\end{array}\right)
\end{aligned}
$$

which is precisely the score of the marginal density of $x_{t}$ with respect to $f_{t}=\left(f_{x, t}, f_{y, t}\right)^{\prime}$.

The result in Theorem 1 substantiates the way missing values are dealt with in papers such as Creal et al. (2014) and others. At the same time, it ties the score-driven modeling approach in the presence of missing value closer to the older and larger literature on how to deal with missing values via expectationmaximization. The alternative argument sketched in Section 2 based on the conditional expectation of $y_{t}$ given $x_{t}$ actually turns out to be false, as the EM approach takes the conditional expectation of the log likelihood function, and not of $y_{t}$ itself.

Also note that if both $x_{t}$ and $y_{t}$ are missing, the EM local objective degenerates to zero, irrespective of $f_{t}$. Also the 'marginal' density degenerates in that case to a constant, such that we obtain $s_{t}^{\mathrm{EM}}=s_{t}^{\mathrm{marg}}=0$. The intuition is clear: if no observations are available containing information on $f_{t}$, then $f_{t}$ cannot be updated and mean reverts.

Interestingly, our result also brings the score-driven approach closer to the treatment of missing values in a state-space framework, where also the marginal score $s_{t}^{\text {marg }}$ is key; see for example Durbin and Koopman (2012). Theorem 1 illustrates that this is fully in line with the score-based approach, and that both approaches are strongly related to the EM approach thanks to the particular features of the score as a propagation mechanism for $f_{t}$. We do note, however, that the assumption of correct specification as used in the proof of Theorem 1 may render the optimality results of Blasques et al. (2015) not applicable to our setting. We leave extensions in this direction for future work.

\section{References}

Blasques, F., S. J. Koopman, and A. Lucas (2015). Information-theoretic optimality of observation-driven time series models for continuous responses. Biometrika 102(2), 325-343.

Bollerslev, T. (1986). Generalized autoregressive conditional heteroskedasticity. Journal of Econometrics 31 (3), $307-327$.

Cox, D. R. (1981). Statistical analysis of time series: some recent developments. Scandinavian Journal of Statistics 8, 93-115.

Creal, D., S. J. Koopman, and A. Lucas (2011). A dynamic multivariate heavy-tailed model for time-varying volatilities and correlations. Journal of Business \& Economic Statistics 29, 552-563.

Creal, D., S. J. Koopman, and A. Lucas (2013). Generalized autoregressive score models with applications. Journal of Applied Econometrics 28(5), 777-795.

Creal, D., S. J. Koopman, A. Lucas, and M. J. Zamojski (2016). Generalized autoregressive methods of moments. Working paper.

Creal, D., B. Schwaab, S. J. Koopman, and A. Lucas (2014). Observation driven mixed-measurement dynamic factor models with an application to credit risk. Review of Economics and Statistics 96(5), 898-915. 
Dempster, A. P., N. M. Laird, and D. B. Rubin (1977). Maximum likelihood from incomplete data via the EM algorithm. Journal of the Royal Statistical Society. Series B (methodological), 1-38.

Durbin, J. and S. J. Koopman (2012). Time series analysis by state space methods. Number 38. Oxford University Press.

Engle, R. F. (1982). Autoregressive conditional heteroscedasticity with estimates of the variance of United Kingdom inflations. Econometrica 50, 987-1008.

Engle, R. F. and G. M. Gallo (2006). A multiple indicators model for volatility using intra-daily data. Journal of Econometrics 131(1), 3-27.

Engle, R. F. and J. R. Russell (1998). Autoregressive conditional duration: a new model for irregularly spaced transaction data. Econometrica 66, 1127-1162.

Harvey, A. C. (2013). Dynamic Models for Volatility and Heavy Tails: with Applications to Financial and Economic Time Series. Econometric Society Monographs. Cambridge: Cambridge University Press.

Harvey, A. C. and A. Luati (2014). Filtering with heavy tails. Journal of the American Statistical Association, forthcoming. Little, R. J. and D. B. Rubin (2014). Statistical analysis with missing data. John Wiley \& Sons.

Oh, D. H. and A. J. Patton (2016). Time-varying systemic risk: Evidence from a dynamic copula model of cds spreads. Journal of Business \& Economic Statistics, to appear.

Opschoor, A., P. Janus, A. Lucas, and D. J. Van Dijk (2014). New heavy models for fat-tailed returns and realized covariance kernels.

Quaedvlieg, R. and P. C. Schotman (2016). Score-driven nelson siegel: Hedging long-term liabilities. Available at SSRN 